\title{
Die Masteralloy-Route als attraktive Legierungsvariante für sinterhärtende Stähle
}

\author{
Christian Gierl-Mayer, Raquel De Oro Calderon und Herbert Danninger
}

Institut für Chemische Technologien und Analytik, TU Wien, Wien, Österreich

Eingegangen 6. Mai 2019; angenommen 12. Juli 2019; online publiziert 31. Juli 2019

Zusammenfassung: Die Möglichkeit, die Eigenschaften von PM-Stählen durch eine Sinterhärtung zu verbessern, hängt von der Menge und der Art der verwendeten Legierungselemente ab. Elemente wie $\mathrm{Cr}$ und $\mathrm{Mn}$ zeigen eine signifikant stärkere Wirkung auf die Härtbarkeit als das bei PMStähle übliche Ni, Cu und Mo. Ihre hohe Sauerstoffaffinität stellt jedoch eine Herausforderung beim Sintern dar. Diese Elemente müssen somit mit anderen Elementen mit geringerer Sauerstoffempfindlichkeit kombiniert werden; z. B. Fe. Dies wird traditionell durch die Verwendung von vorlegierten Pulvern erreicht. Aber auch das Zumischen eines Masterlegierungspulvers zu reinem Eisenpulver stellt eine Alternative dar. Das Masteralloy-Konzept bietet gegenüber der Vorlegierungsroute den Vorteil, dass es eine sehr hohe Flexibilität bei der Auswahl der endgültigen Zusammensetzung des Stahls bietet. Den verschiedenen Arten von Basispulvern können unterschiedliche Mengen an MasterLegierungen beigemischt werden, wodurch sich ein breites Portfolio an Materialeigenschaften ergibt. In dieser Arbeit werden kostengünstige Fe-Mn-Si-Cr-Masterlegierungspulver mit verschiedenen Basispulvern (reines Eisen, MoVorlegierungen und $\mathrm{Cr}$-Vorlegierungen) kombiniert. Die mechanischen Eigenschaften und Mikrostrukturen wurden anhand von Proben ermittelt, die bei $1120^{\circ} \mathrm{C}$ und $1250^{\circ} \mathrm{C}$ gesintert wurden, und zwar sowohl im gesinterten Zustand als auch nach einer anschließenden Wärmebehandlung, die durch Gasabschrecken $\left(\mathrm{N}_{2}\right)$ von $900^{\circ} \mathrm{C}$ (Abkühlgeschwindigkeit von etwa $5^{\circ} \mathrm{C} / \mathrm{s}$ ) erreicht wurde. Die Ergebnisse geben einen hervorragenden Überblick über die verschiedenen Eigenschaften, die erreicht werden können, wenn der Masteralloy-Ansatz verwendet wird, um eine maßgeschneidertes Eigenschaftsprofil zu erzielen. Selbst beim Sintern bei $1120^{\circ} \mathrm{C}$ führen Zusätze von Masterlegierung zu niedrig vorlegierten Basislegierungen zu einer bemerkenswerten Erhöhung der Härte, ohne die Schlagzähigkeit nachteilig zu beeinflussen.

\section{Gierl-Mayer ( $\square)$}

Institut für Chemische Technologien und Analytik,

TU Wien,

Getreidemarkt 9/164-CT,

1060 Wien, Österreich

christian.gierl@tuwien.ac.at
Schlüsselwörter: Niedrigst legierte (lean) PM Stähle, Legierungsvarianten, Masterlegierungen, Mechanische Eigenschaften, Mikrostruktur, Härtbarkeit

The Master Alloy Route as an Attractive Alloy Variant for Sinter-hardening Steels

Abstract: The ability to improve the properties of PM steels by sintering hardening depends on the number and type of alloying elements used. Elements such as $\mathrm{Cr}$ and $\mathrm{Mn}$ show a significantly greater effect on hardenability than the $\mathrm{Ni}$, $\mathrm{Cu}$, and Mo, which are common in PM steels. However, their high oxygen affinity presents a challenge in sintering. Thus, these elements must be combined with other elements of lower oxygen sensitivity, e.g. Fe. This is traditionally achieved through the use of pre-alloyed powders. But adding a master alloy powder to pure iron powder is also an alternative. The master alloy concept has the advantage over the pre-alloy route that it offers very high flexibility in selecting the final composition of the steel. The different types of base powders can be mixed with different amounts of master alloys, resulting in a broad portfolio of material properties. In this work, inexpensive $\mathrm{Fe}-\mathrm{Mn}-\mathrm{Si}-\mathrm{Cr}$ master alloy powders are combined with various base powders (pure iron, Mo and $\mathrm{Cr}$ prealloyed steels). The mechanical properties and microstructures were determined from samples sintered at $1120^{\circ} \mathrm{C}$ and $1250^{\circ} \mathrm{C}$, both in the sintered state and after a subsequent heat treatment reached by gas quenching $\left(\mathrm{N}_{2}\right)$ of $900^{\circ} \mathrm{C}$ (cooling rate of approximately $5^{\circ} \mathrm{C} / \mathrm{s}$ ). The results provide an excellent overview of the various properties that can be achieved when using the master alloy approach to achieve a tailored property profile. Even when sintered at $1120^{\circ} \mathrm{C}$, additions of master alloy to low pre-alloyed base alloys result in a notable increase in hardness without adversely affecting impact strength.

Keywords: Lean PM steels, Alloying variants, Master alloys, Mechanical properties, Microstructure, Hardenability 


\section{Einleitung}

Sinterhärten ist eine attraktive Kombination des Sinter- und des Wärmebehandlungsprozesses, die praktisch in einem Durchgang Präzisionsteile mit attraktiven mechanischen Eigenschaften liefert und, aufgrund der Gasabschreckung, auch keine Nachreinigungsprozesse erfordert, die bei porösen Formteilen ohnehin immer problematisch sind. Die gegenüber z. B. einer Ölhärtung schonendere Gasabschreckung wirkt sich auf die Präzision positiv aus, erfordert jedoch Legierungssysteme, die auch bei der im Sinterofen erreichbaren Kühlrate von 2-3K/s martensitische Gefüge ergeben. Dementsprechend sind höhere Legierungsmetallgehalte als bei ölgehärteten Bauteilen erforderlich.

Dies ist bei den klassischen Legierungssystemen für Sinterformteile problematisch, weil die dort verwendeten Elemente $\mathrm{Cu}, \mathrm{Ni}$ und Mo nicht nur vom Gesundheits- und Umweltaspekt her kritisch sind, sondern auch vom Preisniveau und der Preisstabilität. Höhere Gehalte dieser Legierungselemente erhöhen die Rohstoffkosten und stellen für die Pulvermetallurgie einen Wettbewerbsnachteil gegenüber schmelzmetallugrischen Stählen dar. Aus diesem Grund werden zunehmend auch die in der Schmelzmetallurgie üblichen Legierungselemente $\mathrm{Cr}$, $\mathrm{Mn}$ und $\mathrm{Si}$ für die Pulvermetallurgie untersucht. Allerdings bedeutet die hohe Sauerstoffaffinität dieser Elemente in Kombination mit der großen spezifischen Oberfläche der Pulver für den Sinterprozess, dass sowohl die Entfernung des auf den Pulvern vorhandenen Sauerstoffs schwieriger ist als auch die Anforderungen an die Reinheit der Sinteratmosphäre höher sind als bei $\mathrm{Cu}$-, $\mathrm{Ni}$ - und/oder Mo-legierten Systemen. Als Maßnahme, diese Probleme zumindest zu mildern, bietet sich die Verwendung vorlegierter Pulver an, in denen die genannten Legierungselemente in geringerer Konzentration und damit niedriger chemischer Aktivität vorliegen als bei zugemischten Elementarpulvern; hier sind vor allem Cr- und Cr-Mo-vorlegierte Pulver seit einiger Zeit kommerziell erhältlich. Allerdings ist damit die verfügbare Palette an Zusammensetzungen auf die kommerziell erhältlichen Pulver beschränkt, und die erreichbaren Press- und damit Sinterdichten liegen niedriger als bei unlegierten Pulvern. Um eine Sinterhärtung mit $\mathrm{Cr}$-Vorlegierungen mit geringen Mengen an $\mathrm{Cr}$ (z.B. Fe-1,8Gew\%Cr) zu ermöglichen, werden zur Erhöhung der Festigkeit Zusätze von Ni und $\mathrm{Cu}$ empfohlen.

In der vorliegenden Arbeit wird die sog. MasteralloyTechnik als interessante Alternative präsentiert. In diesem Fall werden alle Legierungsmetalle über ein hochlegiertes Pulver eingebracht, das dem Basispulver - zumeist Reineisen - zugemischt wird. Damit ist für den Formteilhersteller höhere Flexibilität in der Zusammensetzung möglich, und er kann jenen Legierungsgehalt wählen und einstellen, der beim Sinterhärten in seinen Ofenaggregaten zuverlässig martensitisches Gefüge ergibt.

Der Master-Legierungsansatz ermöglicht die Einführung von sauerstoffempfindlichen Elementen wie $\mathrm{Si}, \mathrm{Mn}$ und $\mathrm{Cr}$ [1-4], die die Eigenschaften von niedriglegierten Sinterstählen (z.B. aufgrund der signifikant größeren Wirkung auf die Härtbarkeit von $\mathrm{Cr}$ und $\mathrm{Mn}$ ) im Vergleich zu den traditionellen Legierungselementen, die in klassischen PM-
Stählen verwendet werden, deutlich verbessern können. Außerdem kann die Zusammensetzung der Masterlegierung (Masteralloy $=$ MA) so gestaltet sein, dass die Bildung einer intermediären flüssigen Phase gefördert wird, die das Sintern verbessert [5-11]. Weitere Vorteile des MAVerfahrens sind die Flexibilität bei der Auswahl der endgültigen Zusammensetzung des Stahls (durch einfaches Ändern der MA-Zusätze oder durch Kombination verschiedener Basispulver mit unterschiedlichen MAs) und der im Vergleich zum Vorlegierungsverfahren geringere Effekt auf die Kompressibilität

Die Entwicklung von Masterlegierungen für PM-Stähle ist seit den frühen 1970er Jahren Gegenstand der Forschung. Zu dieser Zeit wurden einige sehr interessante Masterlegierungen mit den Bezeichnungen MCM ( $\mathrm{Mn}-\mathrm{Cr}$ Mo), MVM (Mn-V-Mo) und MM (Mn-Mo) fast zwei Jahrzehnte lang gründlich untersucht, um sie in den USA bei der Produktion von hoch belasteten PM-Teilen einzusetzen [1-3]. Dies wurde jedoch in den 1990er Jahren aufgrund des übermäßigen Werkzeugverschleißes, der durch die sehr harten und scharfkantigen MA-Pulverpartikel (diese wurden zu dieser Zeit durch Gießen und anschließendes Brechen und Mahlen der Blöcke) verursacht wurde, aufgegeben. In den letzten zwei Jahrzehnten hat der Einsatz von Gasverdüsungstechniken die Forschung der MAs vorangetrieben, da damit die Möglichkeit geschaffen wurde, Pulverpartikel mit angepasster Morphologie und niedrigen Sauerstoffgehalten zu erhalten. Das Verfahren liefert jedoch häufig Teilchengrößenverteilungen mit einer mittleren Partikelgröße von 50-100 $\mu \mathrm{m}$, während für viele Anwendungen die Partikelgröße, die erforderlich ist, um eine ausreichende Verteilung der Legierungselemente während des Sinterns sicherzustellen, unter $\sim 25 \mu \mathrm{m}$ liegen sollte. In dieser Arbeit wurden die verwendeten MA-Pulver mit einer neu entwickelten Verdüsungstechnik („Ultra High Pressure Water Atomization (UHPWA)“) hergestellt. Diese MA-Pulver weisen abgerundete Morphologien, niedrige Sauerstoffgehalte $(\sim 0,1 \mathrm{Gew} . \%)$ und geringe Partikelgröße $\left(\mathrm{d}_{50} \sim 8 \mu \mathrm{m}\right)$ bei niedrigen Produktionskosten auf. Dieser Aufsatz gibt einen Überblick über die verschiedenen Eigenschaften, die mit diesen MA-Pulvern in Kombination mit verschiedenen Basispulvern und mit oder ohne zusätzliche Gasabschreckung erhalten werden können.

\section{Experimentelle Durchführung}

Gesinterte Stähle wurden folgendermaßen hergestellt: Basispulver + X Gew.-\% MA+0,5 Gew.-\% C (X=0 Gew.-\%, 4 Gew.-\%, 6 Gew.-\%), wobei die in Tab. 1 beschriebenen Ausgangsmaterialien verwendet wurden. Diese Mischungen wurden bei $600 \mathrm{MPa}$ gepresst und in einem Laborofen mit Silitstabheizung mit gasdichter Superlegierungsmuffel bei $1250^{\circ} \mathrm{C}$ und $1120^{\circ} \mathrm{C}$ für 30 min unter $\mathrm{N}_{2}-5 \mathrm{H}_{2}$-Atmosphäre gesintert. Die Aufheizgeschwindigkeit betrug $\sim 0,5^{\circ} \mathrm{C} / \mathrm{s}$, und die Abkühlgeschwindigkeit für Proben im gesinterten Zustand betrug $\sim 0,75^{\circ} \mathrm{C} / \mathrm{s}$. Einige Proben wurden nach dem Sintern einer Wärmebehandlung unterzogen: Erwärmung auf $900^{\circ} \mathrm{C}$ in $\mathrm{N}_{2}$ für $20 \mathrm{~min}$, gefolgt von Gasabschreckung in $\mathrm{N}_{2}$ mit einer Kühlrate von ungefähr $5^{\circ} \mathrm{C} / \mathrm{s}$. Der Sauer- 


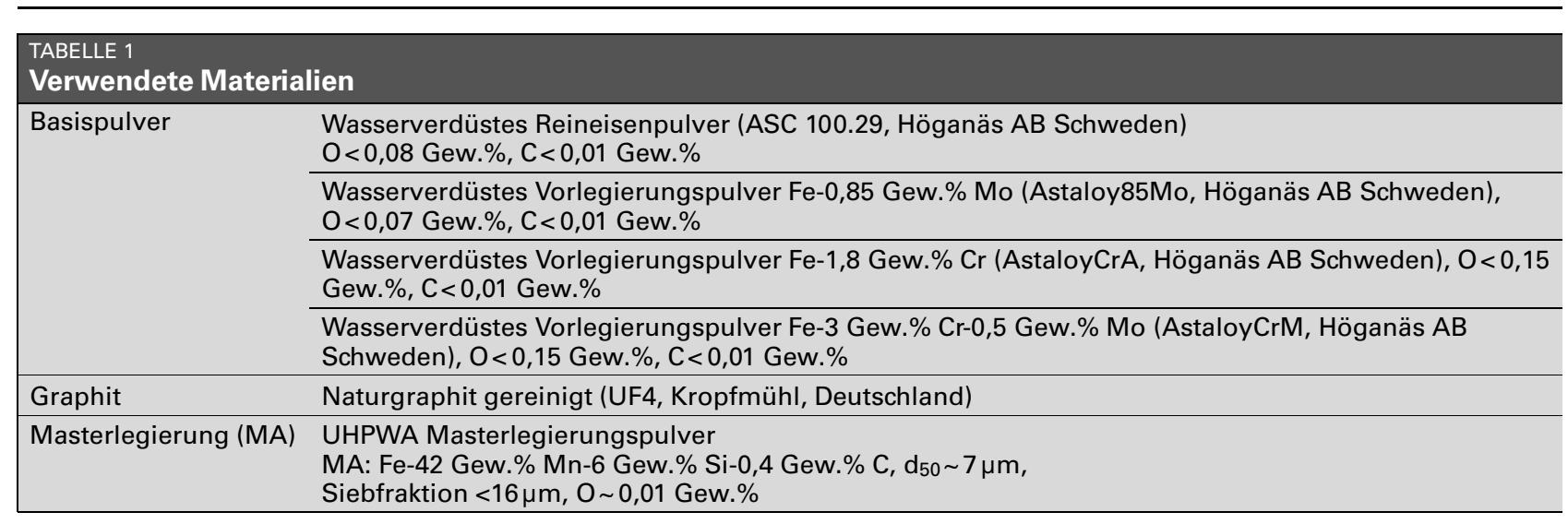

stoff- und Kohlenstoffgehalt der gesinterten Proben wurde unter Verwendung von LECO-TC400 bzw. LECO-CS230 analysiert. Die Schlagzähigkeit, die Mikrohärte und die Makrohärte wurden in Anlehnung an die in ISO 5754: 2017 und ISO 4498: 2010 definierten Standardverfahren getestet.

\section{Ergebnisse und Diskussion}

Die Werte für die Gründichte sind in Abb. 1 angegeben. Die Verpressbarkeit der Basispulver nimmt ab, wenn die Menge der Legierungselemente in den Vorlegierungspulvern ansteigt ( $\mathrm{Fe}>\mathrm{Fe}-0,85 \mathrm{Mo}>\mathrm{Fe}-1,8 \mathrm{Cr}>\mathrm{Fe}-3 \mathrm{Cr}-0,5 \mathrm{Mo})$. Die Zugabe von 4 und 6 Gew.-\% MA zu den Basispulvern entspricht einer Gesamtzugabe von 1,9 Gew.-\% bzw. 2,9 Gew.-\% Legierungselementen über die MA. Durch den Vergleich von Proben mit äquivalenten Zugaben von Legierungselementen, die auf zwei verschiedenen Wegen eingeführt wurden (d. h. Fe+4 MA mit Fe-1,8Cr oder Fe+6MA mit Fe-3Cr), wird die geringere Wirkung von MA-Zugaben auf die Verpressbarkeit gezeigt.

Die Mikrostrukturen von PM-Stählen, die verschiedene MA-Zugaben enthalten, sind in Abb. 2 dargestellt (links 4 Gew.-\% MA und rechts 6 Gew.-\% MA). Geringe Zusätze von MA zu einem Pulver auf Reineisenbasis ergeben bainitische Bereiche, die die im Kern der ursprünglichen Reineisenpulverteilchen vorhandenen perlitisch/ferritischen Mikrostrukturen umgeben. In höher legierten Bereichen werden einige martensitische Bereiche beobachtet, sobald der Zusatz von MA auf 6 Gew.-\% gesteigert wird. In Proben mit
$\mathrm{Fe}+6 \mathrm{MA}$ werden jedoch deutlich höhere Sauerstoffgehalte beobachtet.

Im Fall von Kombinationen mit Fe-0,85Mo-Vorlegierungspulvern werden sehr signifikante Wirkungen mit der Zugabe von nur 4 Gew.-\% MA beobachtet: Die Mikrostruktur besteht aus kleinen Körnern aus oberem Bainit, die von Bereichen mit unterem Bainit und Martensit mit erheblich höherer Härte umgeben sind. Wenn die Zugabe von MA auf 6 Gew.-\% erhöht wird, verschwindet der obere Bainit, und die Kerne aus unterem Bainit sind von breiten martensitischen Bereichen umgeben. Bereits eine Zugabe von 4 Gew.-\% MA bewirkt eine Erhöhung der Makrohärte auf 250 HV10 (von 156 HV10 im Fe.0,85Mo-Basispulver) bei ähnlichen Kohlenstoffgehalten $(\sim 0,35 \%$ C). Die deutlichste Zunahme der Makrohärte wird jedoch beobachtet, wenn Fe-1,8Cr-Vorlegierungspulver verwendet wird: 4 Gew.-\% MA-Zusätze ergeben eine Makrohärte von 310 HV10 im gesinterten Zustand, und 355 HV10 wird mit 6\% MA erhalten (von 141 HV10 im Basispulver). In beiden Fällen besteht die Mikrostruktur aus Kernen aus unterem Bainit, die von martensitischen Bereichen umgeben sind, welche bei Verwendung von $6 \%$ MA-Zusätzen breiter sind.

Abb. 3 zeigt eine Zusammenfassung der Eigenschaften (HV10 gegen Schlagzähigkeit) der verschiedenen Materialien im gesinterten Zustand. Die „Hybrid“-Ansätze (vorlegierte Pulver mit MA-Zusätzen) scheinen die vorteilhaftesten Kombinationen von Eigenschaften zu bieten. Schlagzähigkeit um 20-30 J/cm² und eine Makrohärte um 250-350 HV10 werden im gesinterten Zustand für Stähle mit Kohlenstoffgehalten um 0,30 bis $0,35 \%$ erhalten.
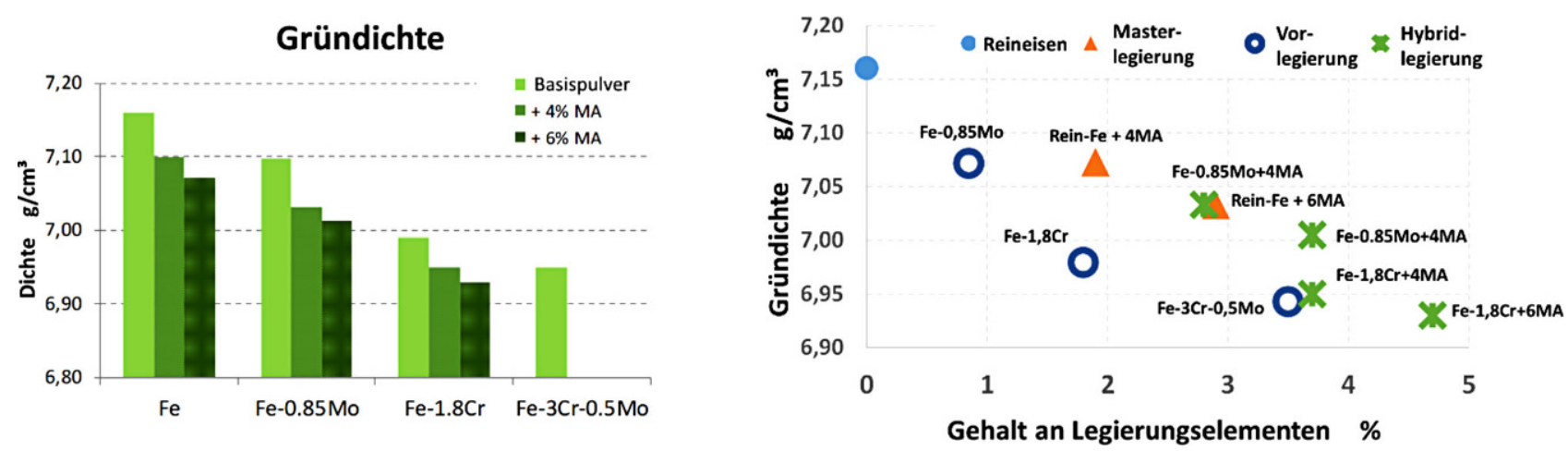

Abb. 1: Gründichten unterschiedlich hergestellter PM-Stähle 
Abb. 2: Mikrostruktur (incl. Mikrohärte der verschiedenen Phasen), Sauerstoff-u. Kohlenstoffgehalt, sowie Makrohärte von PM-Stählen mit Masterlegierungszusätzen von (4 Gew.\% links und $6 \mathrm{Gew}$ \% rechts). gesintert bei $1250^{\circ} \mathrm{C}$, Kühlrate $\sim 0,75^{\circ} \mathrm{C} / \mathrm{s}$. Basispulver:Wasserverdüstes Reineinsen (ASC 100.29): a O: 0,03\%, C: $0,33 \%$, HV $10: 173$, LE: $1,9 \%$, b O: $0,1 \%, C: 0,38 \%$, HV $10: 233$, LE: $2,9 \%$. Basispulver:Wasserverdüstes Vorlegierungspulver $\mathrm{Fe}-0,85 \mathrm{Mo}$ (Astaloy85Mo): c O: $0,02 \%, \mathrm{C}: 0,32 \%$, HV 10 : 250, LE: $2,8 \%$; d O: $0,02 \%$, C $0,32 \%$, HV10: 300 , LE: $3,7 \%$. Basispulver: Wasserverdüstes Vorlegierungspulver $\mathrm{Fe}-1,8 \mathrm{Cr}$ (AstaloyCrA): e O: 0,03\%, C: $0,33 \%$, HV10: 310 , LE: $3,7 \%$ fO: $0,03 \%, C: 0,33 \%$, HV $10: 355$, LE: $4,7 \%$

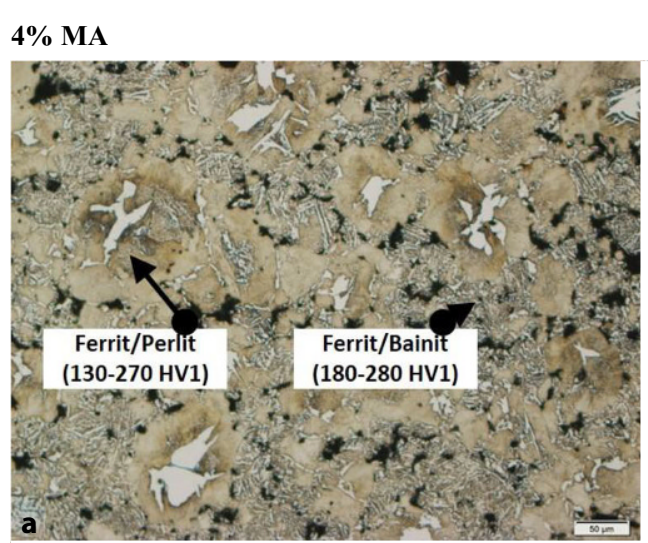

6\% MA
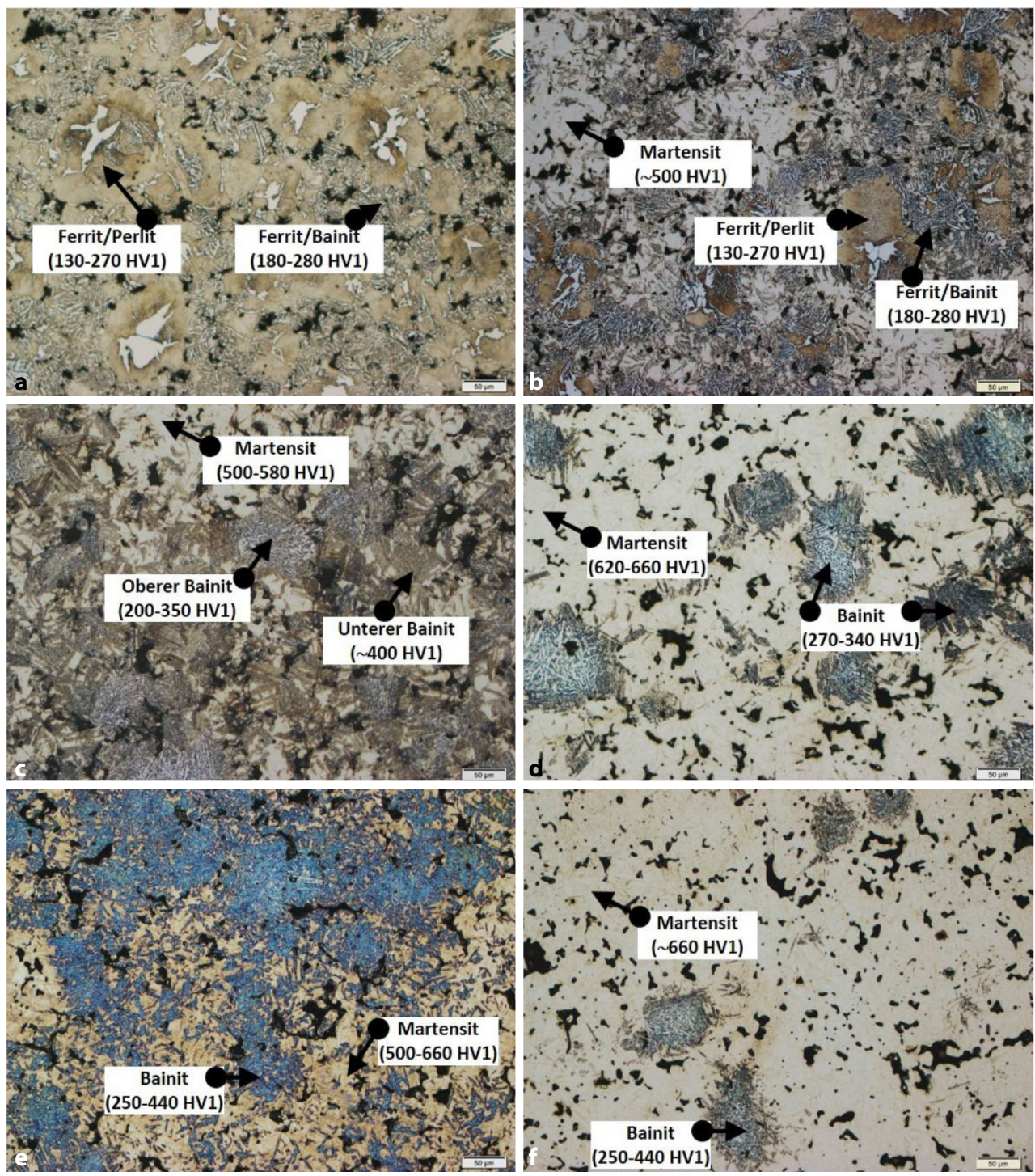

Abb. 4 zeigt die Ergebnisse, die bei Proben erhalten wurden, die bei $1120^{\circ} \mathrm{C}$ gesintert wurden, und schließt die Eigenschaften von Proben ein, die einer Gasabschreckung in $\mathrm{N}_{2}$ unterzogen wurden, um eine geschätzte Abkühlgeschwindigkeit von etwa $5 \mathrm{~K} / \mathrm{s}$ zu erhalten. Nach diesem Wärmebehandlungsprozess wurde keine Anlassbehandlung durchgeführt. Die Ergebnisse können einfacher analysiert werden, wenn Kombinationen betrachtet werden, die eine äquivalente Gesamtmenge an Legierungselementen ergeben. Für Gesamtzugaben an Legierungselementen unter $2 \mathrm{Gew} .-\%$ ( $\mathrm{Fe}+4 \mathrm{MA}$, Fe-1,85Mo und $\mathrm{Fe}-1,8 \mathrm{Cr}$ ) liegen die erhaltenen Härtewerte unter 200 HV10. Kombinationen von vorlegierten Pulvern mit nur $4 \mathrm{Gew}$ \% MA (Fe$0,85 \mathrm{Mo}+4 \mathrm{MA}$ und $\mathrm{Fe}-1,8 \mathrm{Cr}+4 \mathrm{MA}$ mit einem Gesamtlegierungsgehalt von 2,7 und 3,7 Gew.\%) ergeben jedoch eine Härte von 250 HV10 bei einer Schlagzähigkeit ähnlich den Basispulvern. Im Fall von $\mathrm{Fe}-1,8 \mathrm{Cr}+4 \mathrm{MA}$ kann die Härte mit einem Sinterhärtungsschritt auf $\sim 350$ HV10 erhöht werden, ohne die Schlagzähigkeit zu beeinträchtigen.
Die Vorteile der Zumischung von geringen Mengen MA zu vorlegierten Pulversorten wird bei einer Sinterung bei $1250^{\circ} \mathrm{C}$ (Abb. 5) noch deutlicher. Wenn die Eigenschaften der Proben $\mathrm{Fe}-0,85 \mathrm{Mo}+4 \mathrm{MA}$ und $\mathrm{Fe}-1,8 \mathrm{Cr}+4 \mathrm{MA}$ mit denen von $\mathrm{Fe}-3 \mathrm{Cr}-0,5 \mathrm{Mo}$ verglichen werden (mit einem ähnlichen Legierungsgehalt, jedoch durch einen vorlegierten Ansatz eingeführt), wird beobachtet, dass Hybridkombinationen eine höhere Härte und höhere Schlagzähigkeiten ergeben. Eine weitere Erhöhung der Härte der Hybridqualitäten kann durch Gasabschreckung in den Gemischen mit 4\% MA oder durch Erhöhung der Zugaben von Masterlegierung auf $6 \mathrm{Gew}$.-\% erzielt werden. In beiden Fällen sind die Werte der Schlagzähigkeit in den bei $1250^{\circ} \mathrm{C}$ gesinterten Proben immer noch höher als diejenigen, die mit dem vollständig vorlegierten Pulver erhalten wurden.

Abb. 6 zeigt einen Vergleich der Eigenschaften (Härte gegen Schlagzähigkeit) für Stähle im gesinterten Zustand, die bei $1120^{\circ} \mathrm{C}$ oder $1250^{\circ} \mathrm{C}$ gesintert wurden. Die gestrichelten Kreise begrenzen zwei Materialfamilien: eine mit Legie- 


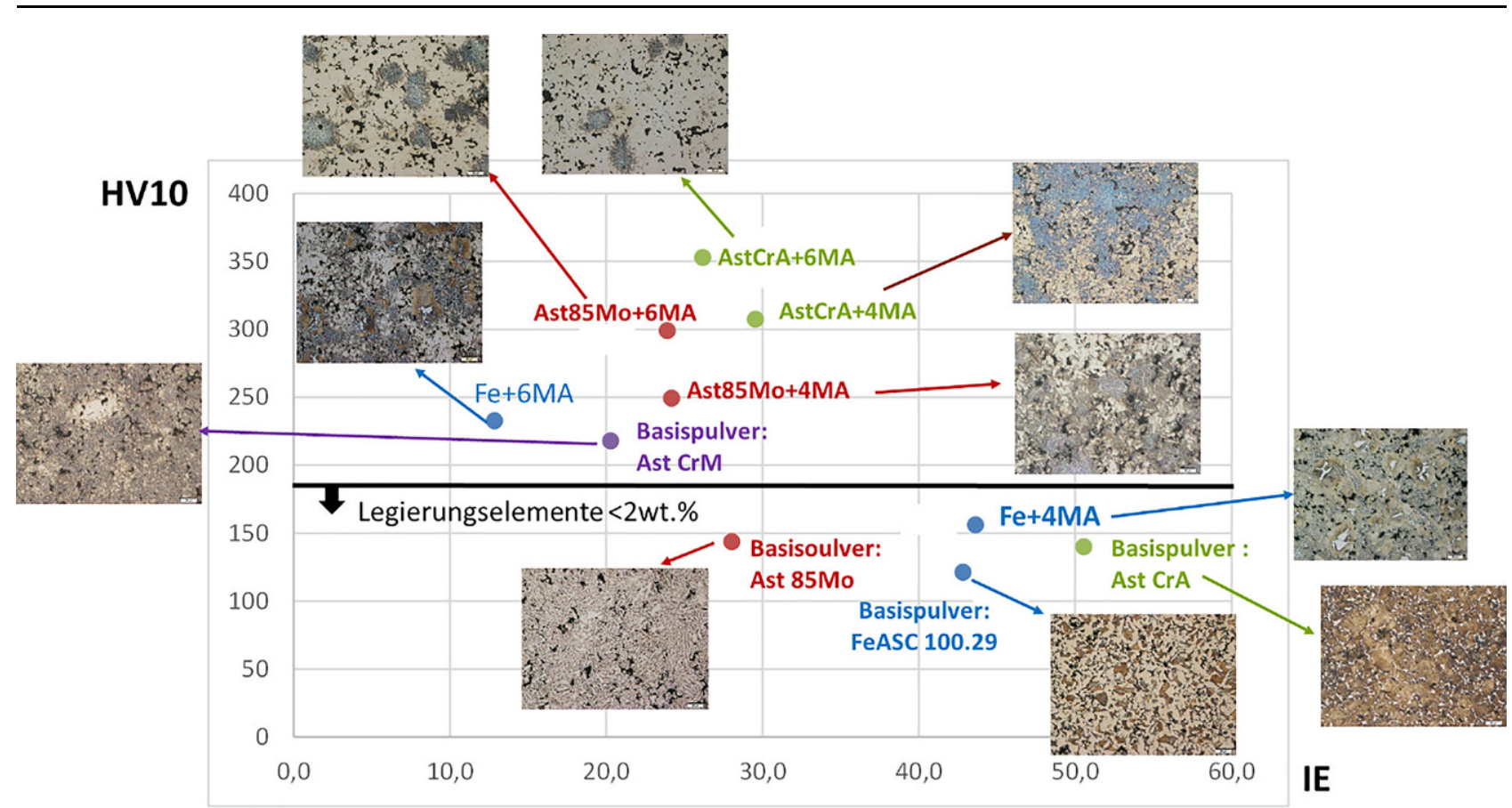

Abb. 3: Makrohärte (HV10) gegen Schlagzähigkeit $\left(\mathrm{J} / \mathrm{cm}^{2}\right)$ von PM-Stählen, im Sinterzustand; gesintert bei $1250^{\circ} \mathrm{C}$
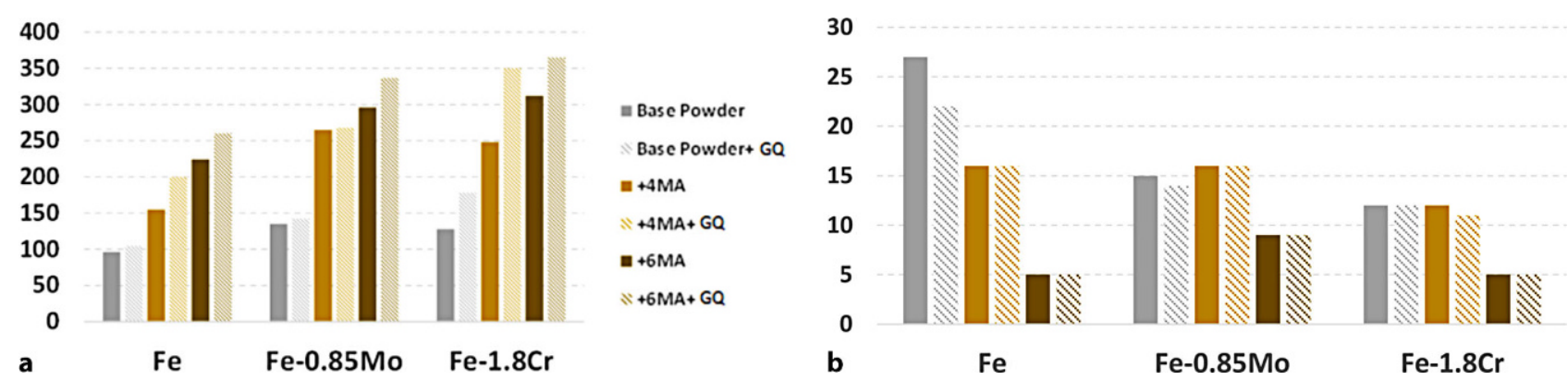

a

$\mathrm{Fe}$

Fe-0.85Mo Fe-1.8Cr

b

$\mathrm{Fe}$

Fe-0.85Mo

Fe-1.8Cr

Abb. 4: a, b Makrohärte ( $\mathrm{HV} 10)$ und Schlagzähigkeit $\left(\mathrm{J} / \mathrm{cm}^{2}\right)$ von PM-Stählen gesintert bei $1120^{\circ} \mathrm{C}$, im Sinterzustand und nach Gasabschreckung in $\mathrm{N}_{2}$ (GQ)

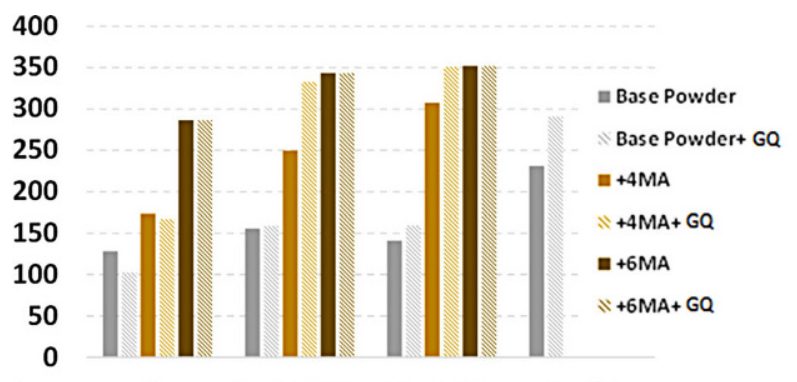

a
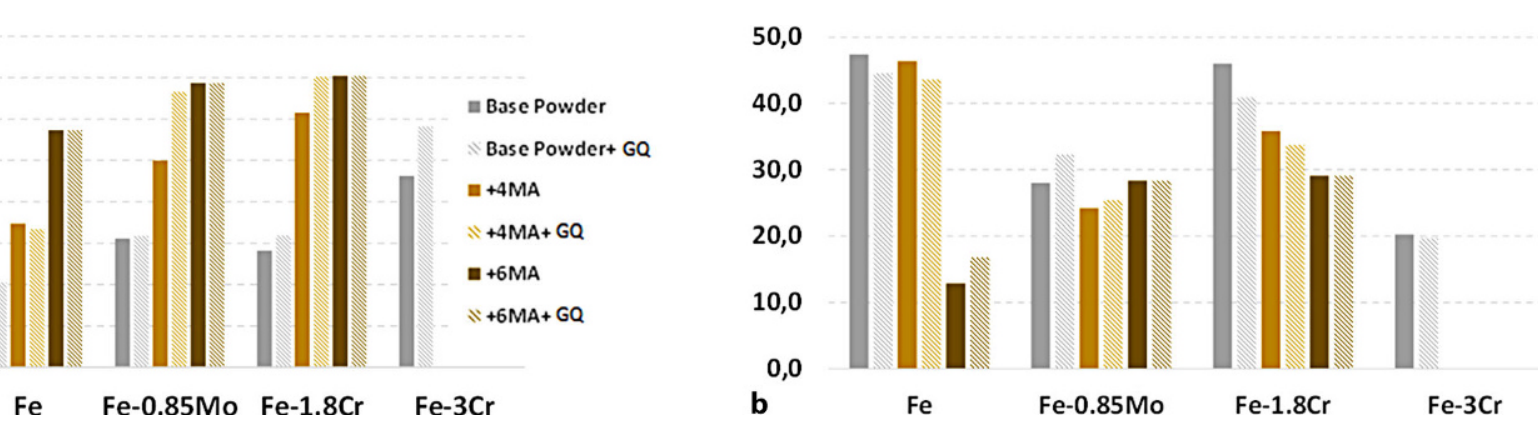

Abb. 5: a, b Makrohärte (HV10) und Schlagzähigkeit $\left(\mathrm{J} / \mathrm{cm}^{2}\right)$ von PM-Stählen gesintert bei $1250^{\circ} \mathrm{C}$, im Sinterzustand und nach Gasabschreckung in $\mathrm{N}_{2}$ (GQ)

rungsgehalten unter $2 \mathrm{Gew} .-\%$ und eine andere mit Legierungsgehalten um 3 bis $4 \mathrm{Gew} .-\%$. Blaue Markierungen stehen für bei $1120^{\circ} \mathrm{C}$ gesinterte Stähle und rote Markierstähle für $1250^{\circ} \mathrm{C}$.

In der Gruppe der Stähle mit Legierungsanteilen unter 2 Gew.-\% ist es möglich, die Einführung von Legierungselementen entweder mit einem vorlegierten Ansatz (PA
$\mathrm{Fe}-1,8 \mathrm{Cr}$ ) oder mit einer Masterlegierung (Rein-Fe+4MA) zu vergleichen. Bei ähnlichem Legierungsgehalt sind die Eigenschaften von MA enthaltenden Stählen denen, die mit dem vorlegierten Pulver erhalten werden, immer leicht überlegen.

In der Gruppe der Stähle mit 3 bis 4 Gew.-\% Legierungselementen ist das Verhalten von Hybridkombinationen 
Abb. 6: Makrohärte gegen Schlagzähigkeit von Stählen unterschiedlicher Herstellungsrouten. Reiner Masteralloy Ansatz: Reines $\mathrm{Fe}+4 \mathrm{MA}$, Vorlegierungsansatz: $\mathrm{Fe}-1,8 \mathrm{Cr}$ oder Fe-1,8Cr-0,5Mo, Hybridansatz: $\mathrm{Fe}-1,8 \mathrm{Cr}+4 \mathrm{MA}$ und Fe-0,5Mo + 4MA. Die Zusammensetzung der Masterlegierung ist Fe-42Mn-6Si-0,4C

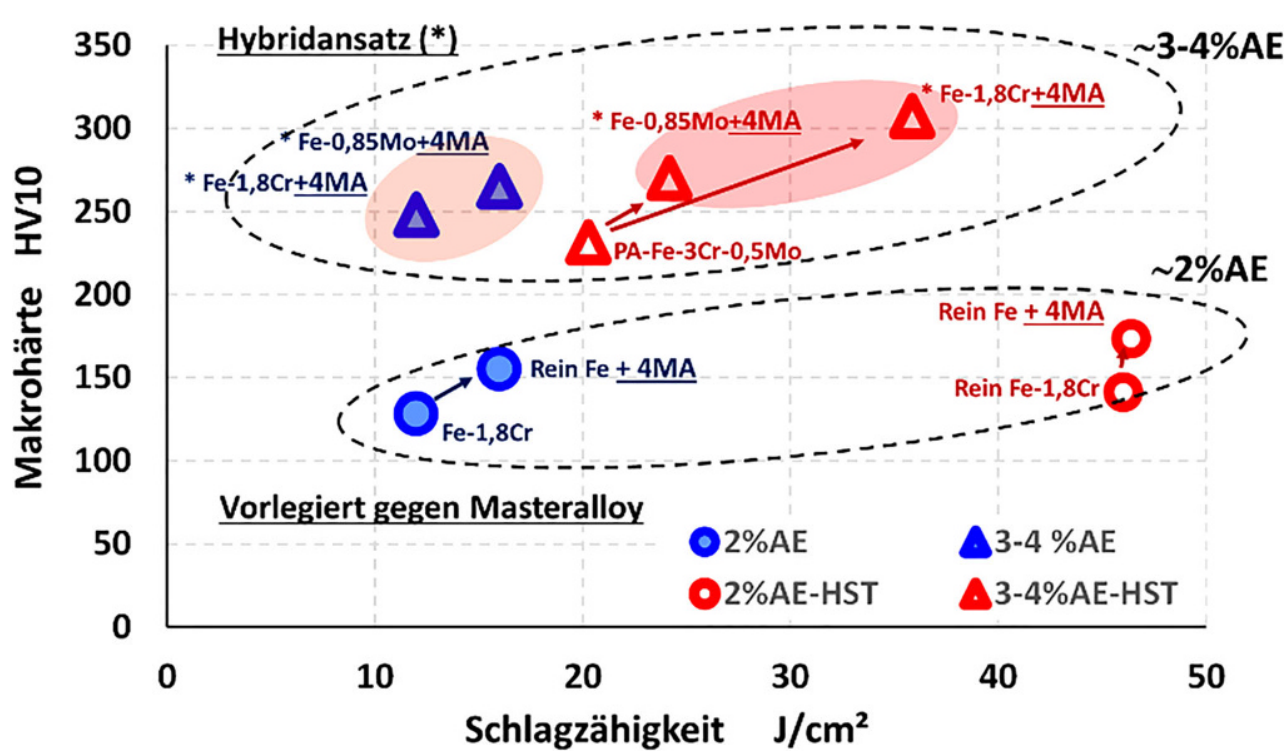

traktiver ist. Bei den niedrigeren Sintertemperaturen zeigt der in dieser Arbeit vorgestellte "Hybrid“-Ansatz (Kombination von Masterlegierungen mit vorlegierten Pulvern mit geringen Legierungselementgehalten: $\mathrm{Fe}-0,85 \mathrm{Mo}$ und $\mathrm{Fe}-$ $1,8 \mathrm{Cr}$ ) sehr interessante Eigenschaftskombinationen (Härte zwischen 250-350 HV10 kombiniert mit Schlagzähigkeiten $\left(10-15 \mathrm{~J} / \mathrm{cm}^{2}\right.$ für Stähle mit Kohlenstoffendgehalten um $0,4 \%)$ ). Bei Proben, die bei $1250^{\circ} \mathrm{C}$ gesintert werden, weisen die Hybridkombinationen eine höhere Härte und höhere Schlagzähigkeit auf als mit einer ähnlichen Menge an Legierungselementen, die durch einen vorlegierten Ansatz eingeführt wurden (d. h. mit Fe-3Cr-0,5Mo-Vorlegierungspulvern). Beim Sintern dieser Hybridkombinationen bei $1250^{\circ} \mathrm{C}$ ist es möglich, die Härte entweder durch Sinterhärtung oder mit einer Erhöhung des MA-Gehaltes zu erhöhen. Die Menge an MA (bis zu 6\% MA) hat keinen drastisch negativen Einfluss auf die Schagzähigkeit. Die MA-Route kann somit als wirksames Werkzeug zum "Anpassen“ der Leistung von PM-Material verwendet werden. Durch einfaches Anpassen der zugesetzten MA-Menge oder durch Kombinieren des MA-Pulvers mit verschiedenen Basispulvern (reines Eisen oder vorlegierte Qualitäten) können die Eigenschaften von Sinterstählen in einem weiten Rahmen variiert und an die Anforderungen angepasst werden.

Funding. Open access funding provided by TU Wien (TUW).

Open Access Dieser Artikel wird unter der Creative Commons Namensnennung 4.0 International Lizenz (http://creativecommons.org/licenses/ by/4.0/deed.de) veröffentlicht, welche die Nutzung, Vervielfältigung, Bearbeitung, Verbreitung und Wiedergabe in jeglichem Medium und Format erlaubt, sofern Sie den/die ursprünglichen Autor(en) und die Quelle ordnungsgemäß nennen, einen Link zur Creative Commons Lizenz beifügen und angeben, ob Änderungen vorgenommen wurden.

\section{Literatur}

1. Zapf, G.; Dalal, K.: Introduction of high oxygen affinity elements manganese, chromium, and vanadium in the powder metallurgy of beim Sintern bei $1250^{\circ} \mathrm{C}$ besonders auffällig, aber auch beim Sintern bei $1120^{\circ} \mathrm{C}$ offensichtlich, was industriell at- 
P/M parts, Modern developments in powder metallurgy, 10 (1977), S. $129-152$

2. Schlieper, G.; Thümmler, F.: High strength heat-treatable sintered steels containing manganese, chromium, vanadium and molybdenum, Powder Metallurgy International, 11 (1979), S. 172-176

3. Banerjee, S.; Schlieper, G.; Thümmler, F.; Zapf, G.: New Results in the Master Alloy Concept for High Strength Sintered Steels, Progress in Powder Metallurgy, 13 (1980), S. 143-157

4. Beiss, P.: Alloy Cost Optimization of High Strength Mn-Cr-Mo Steels with Kerosene Atomized Master Alloy, Advances in Powder Metallurgy \& Particulate Material, 1 (2006), S. 12-20

5. Fischmeister, H. F.; Larsson, L. E.: Fast diffusion alloying for powder forging using a liquid phase, Powder Metallurgy, 17 (1974), S. 227-240

6. Mocarski, S.; Hall, D. W.; Chernenkoff, R. A.; Yeager, D. A.; McHugh, C. O.: Master alloys to obtain premixed hardenable powder metallurgy steels, Powder Metallurgy, 39 (1996), S. 130-137

7. Klein, A. N.; Oberacker, R.; Thümmler, F.: Development of new high strength $\mathrm{Si}-\mathrm{Mn}$-alloyed sintered steels, Modern Developments in Powder Metallurgy, 16 (1985), S. 141-152
8. Tojal, C.; Gomez-Acebo, T.; Catro, F: Development of PM Stainless Steels with Improved Properties through Liquid Phase Sintering, Materials Science Forum, 534-536 (2007), pp 661-664

9. Castro, F; Sarasola, M; Sainz, S, Gomez-Acebo, T. : Processing routes for obtaining novel high performance Mn-containing PM steels, Progress in Powder Metallurgy, Pts 1 and 2, 2007, S. 534-536 und S. 705-708

10. Oro, R.; Campos, M.; Torralba, J. M.; Capdevila, C.: Lean alloys in PM: from design to sintering performanceet al., Powder Metallurgy, 55 (2012), S. 294-301

11. De Oro Calderón, R.; Bernardo, E.; Campos, M.; Gierl-Mayer, C.; Danninger, H.; Torralba, J. M.: Tailoring master alloys for liquid phase sintering: Effect of introducing oxidation-sensitive elements.et al., Powder Metallurgy, 59 (2016), S. 31-40

Hinweis des Verlags. Der Verlag bleibt in Hinblick auf geografische Zuordnungen und Gebietsbezeichnungen in veröffentlichten Karten und Institutsadressen neutral. 